\section{Ernährung: Auch Senioren snacken gern}

Ob gesund oder nicht: Der kleine Happen zwischendurch statt einer Hauptmahlzeit steht hoch im Trend - obwohl $67 \%$ der über 4000 für eine Studie Befragten angaben, dass Zeit und Ruhe beim Essen wichtiger seien als Abwechslung, Frische oder Gemeinsamkeit. Nestlé-Studie 2011

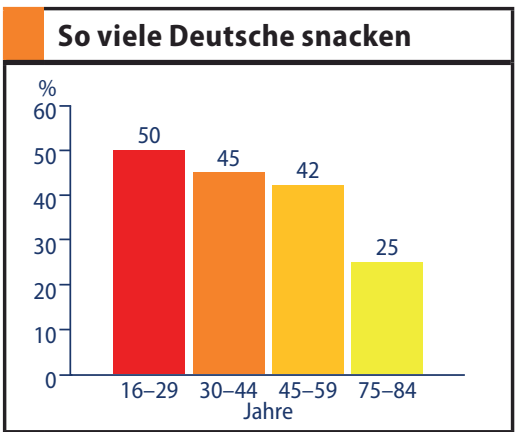

\section{Notfall: Kinder leisten kompetent Erste Hilfe}

Schon Vorschulkinder sind in der Lage, die Bewusstlosigkeit eines Unfallopfers richtig einzuschätzen oder sich die Notrufnummer zu merken, so das Resultat einer Untersuchung mit zehn Vier- bis Fünfjährigen. Die Knirpse handelten nach Teilnahme an einem Erste-Hilfe-Kurs auch noch zwei Monate später überraschend kompetent. Somit sei der frühe Beginn von Erste-Hilfe-Trainings wichtig und sinnvoll, bekräftigte der Studienleiter.

Bollig G et al, Scand J Trauma Resusc Emerg Med 2011 (online first)

\section{SpringerMedizin via Twitter folgen}

Der Frühling hält Einzug und das Zwitschern wird immer lauter: Werden Sie zum "Follower" und "lauschen" Sie uns bei Twitter.

\section{Die weite Welt der Prävention} HDL und „Pille“ halten
Darmkrebs in Schach
Frauen, die regelmäßig orale Kontrazeptiva einnehmen oder hohe HDL-Konzentrationen im Blut haben, schützen ihren Darm vor Krebs. Letzteres gilt auch für Männer.

Eine Langzeitstudie mit über 520.000 Teilnehmern untersuchte in zehn europäischen Ländern die Effekte der Ernährung auf die Krebsentstehung. Ergebnis: Je höher die HDL- und Apolipoprotein A (Apo A)-Konzentrationen im Blut waren, desto geringer war das Risiko, an einem Kolonkarzinom zu erkranken. Dabei blieb die präventive Wirkung auf das Kolon beschränkt; das Rektumkarzinomrisiko wurde weder durch Apo A noch durch HDL beeinflusst.

In der Studie befragten Wissenschaftler die Teilnehmer nach ihren Ernährungsgewohnheiten und werteten Blutproben von Karzinompatienten und gesunden Probanden aus. Sie verglichen jeweils Teilnehmer gleicher Nationalität und gleichen Alters.

\section{Welche Rolle spielen Östrogene?}

Ein anderer potenziell schützender Faktor vor Darmkrebs scheint die regelmäßige Einnahme von oralen Kontrazeptiva zu sein. In einer Untersuchung mit 337.802 Frauen im Alter zwischen 35 und 70 Jahren zeigte sich ein vermindertes Dickdarmkrebsrisiko bei denjenigen, die in ihrem Leben jemals „die Pille“ geschluckt hatten. Hier schien der Schutz zwar nicht auf das Kolon beschränkt, doch sind weitere Analysen für die Klärung des Kausalzusammenhangs gefordert.

\section{Hoffnung für Diabetiker Insulintherapie: Dreimal pro Woche spritzen reicht!}

Wird das Gepikse mit dem InsulinPen immer seltener nötig? Laut Studie senkt ein neues Insulinanalogon den Blutzucker genauso effektiv wie bereits zugelassene Präparate - und muss nur dreimal wöchentlich appliziert werden.

Nach Injektion des Ultralangzeit-Insulins degludec in das subkutane Fettgewebe bilden sich dort sogenannte "Multihexamere“, die langsam und zeitversetzt die einzelnen Insulinmoleküle freisetzen. So erklärt sich die Depotwirkung, sowie die Tatsache, dass das Präparat nur drei mal pro Woche - statt wie bisher täglich - von Diabetikern gespritzt werden muss.

In einer Vergleichsstudie mit 245 Typ2-Diabetikern stellte sich heraus, dass Insulin degludec in Bezug auf die $\mathrm{HbA}_{1 c}-$ Senkung einem täglich gespritzten Langzeitinsulin (Insulin glargin) ebenbürtig ist. Allerdings kam es unter dem neuen Medikament häufiger zu einem Abfall des Blutzuckers auf unter $3,1 \mathrm{mmol} / \mathrm{l}$. Weitere Untersuchungen müssen deshalb klären, ob Insulin degludec in Zukunft zur Anwendung zugelassen wird. 